\title{
LA EXIGENCIA DE RESPONSABILIDAD A LAS EMPRESAS POR LAS VIOLACIONES DE DERECHOS HUMANOS RELACIONADAS CON EL LAND GRABBING: EVOLUCIÓN Y PERSPECTIVAS
}

\author{
Marco FAsciglione*
}

El land grabbing, también conocido como «acaparamiento de tierras», se ha convertido en un área de creciente preocupación debido a su impacto en una serie de derechos humanos fundamentales, razón por la cual en la actualidad se ha vuelto a prestar atención a la forma en la que se distribuye, utiliza, controla y gestiona la tierra. En este contexto, cada vez se otorga mayor atención a las responsabilidades en materia de derechos humanos de las empresas comerciales, actores influyentes en la gobernanza de la tierra y de otros recursos naturales de manera conjunta con los Estados. En efecto, cuando las empresas expresan su interés en adquirir derechos de uso o propiedad a largo plazo sobre tierras y bosques (o cuando un gobierno solicita inversiones de empresas en su país), con frecuencia se negocian y celebran acuerdos que pasan por alto los derechos de los usuarios y propietarios locales de la tierra. Las minorías, pueblos indígenas y comunidades que utilizan la tierra y los bosques, a menudo quedan fuera de las negociaciones entre las empresas y los gobiernos, sentando así las bases para nuevos conflictos o para la reavivación de los ya existentes.

El presente comentario ofrecerá una breve panorámica de las normas internacionales aplicables desde una perspectiva de los derechos humanos a las empresas que participan en adquisiciones y arrendamientos de tierras a

* El autor es investigador del Consejo Nacional de Investigaciones (CNR, Nápoles) en Derecho internacional, en donde es el Investigador Principal del proyecto Corporate human rights and environmental due diligence and the Promotion of Corporate Responsibility (CO.RE) y Codirector de la Escuela Internacional de Verano en Empresas y Derechos Humanos. Además, es Jefe del Comité Editorial de la revista Diritti umani e diritto internazionale. Previamente, fue funcionario jurídico en la Corte Europea de Derechos Humanos (m.fasciglione@iriss.cnr.it). «Esta publicación es el resultado de las actividades de investigación realizadas en el marco del Proyecto CLIC - Circular models Leveraging Investments in Cultural heritage adaptive reuse, financiado por H2020». 
gran escala. Estas normas pertenecen al ámbito intelectualmente desafiante - y aún en desarrollo- conocido como "empresas y derechos humanos» ${ }^{1}$.

Resulta evidente que actualmente el Derecho internacional de los derechos humanos aún no ha previsto un derecho humano universal a la tierra, y la regulación de la mayoría de las cuestiones relativas a la misma es dejada en manos del Derecho nacional. No obstante, debe señalarse que ciertos instrumentos internacionales han encontrado la manera de conectar la tierra con el disfrute de los derechos humanos. Por ejemplo, el párr. 2 del art. 11 del Pacto Internacional de Derechos Económicos, Sociales y Culturales y el apdo. g) del párr. 2 del art. 14 de la Convención sobre la eliminación de todas las formas de discriminación contra la mujer hacen referencia a la tierra en relación con el derecho a la alimentación y a los derechos de la mujer rural, respectivamente. De manera similar, los instrumentos jurídicos en materia de derechos humanos ofrecen una clara protección a ciertos grupos. Los pueblos indígenas, por ejemplo, tienen un derecho humano a la tierra, protegido por el Convenio 169 de la OIT sobre Pueblos Indígenas y Tribales de 1989 y la Declaración de las Naciones Unidas sobre los Derechos de los Pueblos Indígenas de 2007. Además, el derecho a la tierra de los pueblos indígenas ha sido ampliamente interpretado en relación con otros tratados por tribunales de derechos humanos y órganos de control, como el Pacto Internacional de Derechos Económicos, Sociales y Culturales, hasta el punto de extender este derecho también a ciertos grupos no indígenas, como las comunidades tribales.

En la actualidad, un conjunto de principios de autoridad reconocida que hacen frente al impacto negativo en los derechos humanos generado por la puesta en marcha de regímenes de land grabbing por parte de actores empresariales están consagrados en los «Principios Rectores de las Naciones Unidas sobre las Empresas y los Derechos Humanos» (en adelante, los «Principios Rectores»), adoptados por el Consejo de Derechos Humanos de la ONU en 2011. A pesar de su naturaleza no vinculante, la esencia de los Principios Rectores es, de hecho, la distinción entre el deber de los Estados de proteger y la responsabilidad empresarial de respetar los derechos humanos, así como su contribución con la concretización del derecho de acceso de las víctimas a un recurso efectivo. ¿Qué nos dicen los Principios Rectores en cuanto al impacto negativo sobre los derechos humanos originado por las prácticas corporativas de land grabbing?

En lo que respecta al deber de los Estados de proteger, los Principios Rectores reflejan una serie de reglas ya existentes bajo el Derecho internacional, recordando que los Estados, en su calidad de principales responsables, tienen la obligación de respetar, proteger y hacer efectivos los derechos humanos de las personas que se encuentran en su jurisdicción frente a las violaciones cometidas por empresas comerciales ${ }^{2}$. En consecuencia, los Estados tienen

1 Véase Fasciglione, M., Diritti umani e responsabilità sociale d'impresa nel diritto internazionale, Nápoles, Editoriale Scientifica 2010; Marullo, M. C. y Zamora CABOT, F. J., Empresas y derechos humanos. Temas Actuales, Nápoles, Editoriale Scientifica, 2018.

2 Véase el Principio 1 de los Principios Rectores. 
dos tipos de obligaciones. Una obligación negativa de respetar los derechos humanos en relación con las empresas que actúan como agentes del Estado (las empresas estatales son un ejemplo típico); y una obligación positiva de garantizar los derechos humanos de las personas bajo su jurisdicción, adoptando todas las medidas razonables y apropiadas para prevenir y remediar las violaciones perpetradas por las empresas comerciales ${ }^{3}$. Cuando se trata de obligaciones negativas, la existencia de un nexo estricto entre el Estado y la empresa (p. ej., una relación de propiedad o de control) añade otro nivel de responsabilidad y puede aplicarse la norma secundaria, por tanto, los Estados pueden ser considerados jurídicamente responsables de los comportamientos de esas entidades ${ }^{4}$. En lo que respecta a las obligaciones positivas, el deber del Estado de proteger exige que organice y utilice sus facultades normativas y de política para regular también las conductas del sector privado. Los órganos creados en virtud de tratados de derechos humanos han interpretado que esta obligación tiene consecuencias tanto para los Estados anfitriones (en donde opera una empresa multinacional) como para los Estados de origen (en donde una empresa multinacional tiene su sede o está constituida) de las compañías multinacionales involucradas en controversias sobre land grabbing. La jurisprudencia de la Corte Interamericana de Derechos Humanos (CIDH) es muy instructiva sobre cómo se aplican estas obligaciones positivas a los Estados anfitriones, especialmente, en relación con el impacto adverso de las operaciones de la industria extractiva sobre las tierras indígenas. En efecto, la CIDH ha interpretado este deber como una obligación de prevenir $^{5}$, determinar e investigar ${ }^{6}$, así como de remediar $^{7}$ las violaciones de los derechos humanos relacionadas con la explotación de tierras por parte de las empresas; y ha instado a los Estados a mantener un «espacio normativo nacional» para la tutela de los derechos humanos en acuerdos internacionales sobre inversiones y transacciones en materia de tierras ${ }^{8}$.

Desde la segunda perspectiva, el Estado de origen de una empresa comercial mantiene también cierta responsabilidad de regular la conducta de sus empresas en todas sus operaciones mundiales. Las Directrices voluntarias

3 En lo que se refiere específicamente al sistema regional europeo, véase FASCigLione, M., «Enforcing the State Duty to Protect under the UN Guiding Principles on Business and Human Rights: Strasbourg views», en Bonfanti, A. (ed.), Business and Human Rights in Europe: International Law Challenges, Nueva York y Londres, Routledge, 2018, pp. 37-47.

4 Muchas empresas estatales, de hecho, están asociadas al impacto negativo de los derechos humanos que se derivan de las operaciones de compraventa de tierras. Véase Consejo de Derechos Humanos, Report of the Working Group on the issue of human rights and transnational corporations and other business enterprises, UN Doc. A/HRC/32/45, de 4 de mayo de 2016.

5 Véase Corte Interamericana de Derechos Humanos, Pueblos Kaliña y Lokono c. Surinam, Sentencia de 25 de noviembre de 2015, párrs. 221-224.

6 Véase Corte Interamericana de Derechos Humanos, Pueblo Indígena Kichwa of Sarayaku c. Ecuador, Sentencia de 27 de junio de 2012, párrs. 265, 271.

7 Véase Pueblos Kaliña y Lokono c. Surinam, cit., nota 5, párrs. 237-239.

8 Véase el Principio 9 de los Principios Rectores. Esta cuestión está, por tanto, estrechamente ligada al debate actual sobre el principio de soberanía en el marco del Derecho internacional. Véase la contribución de apertura de este foro y también NiNO, M., Land grabbing e sovranità territoriale in diritto internazionale, Nápoles, Editoriale Scientifica, 2018. 
sobra la gobernanza responsable de la tenencia de la FAO, de 2012, que incorporan los Principios Rectores, hacen hincapié en que "cuando se trate de sociedades transnacionales, los Estados de origen tienen un papel que desempeñar para ayudar tanto a las empresas como a los Estados de acogida con el fin de asegurar que las empresas no estén involucradas en abusos contra los derechos humanos y los derechos legítimos de tenencia» (directriz 3.2). Los Organismos de control para la protección de derechos humanos han confirmado esta opinión, adoptando un enfoque amplio en cuanto al alcance territorial del deber del Estado de proteger los derechos humanos contra las violaciones que se producen fuera de su jurisdicción como consecuencia de las actividades de las entidades comerciales sobre las que pueden ejercer control; esto, especialmente, en los casos en que los recursos de que disponen las víctimas ante los tribunales nacionales del Estado en que se produce el daño no están disponibles o son ineficaces ${ }^{9}$. Sin embargo, en virtud de las normas internacionales de derechos humanos, se pide a los Estados que adopten medidas conjuntas e independientes para lograr la cooperación internacional en la solución de problemas de carácter económico, social, cultural o humanitario; y en la promoción y el fomento del respeto de los derechos humanos. También deben velar por que la conducta de las organizaciones multilaterales de las que son miembros, incluidas las organizaciones financieras o comerciales internacionales, no tengan consecuencias negativas para los derechos humanos relacionados con la tierra ${ }^{10}$.

En lo que respecta a las empresas, bajo los Principios Rectores, las compañías nacionales y transnacionales que participan en operaciones de compraventa de tierras, inversiones y otras actividades que involucren la adquisición, utilización o modificación de tierras, tienen la responsabilidad de no vulnerar los derechos de otros usuarios y propietarios y de hacer frente a cualquier efecto adverso que surja como resultado de sus actividades. Aquí se aplica el segundo pilar de los Principios Rectores. La responsabilidad de las empresas de respetar los derechos humanos es una norma global de conducta esperada de todas las empresas y existe independientemente de la capacidad o la voluntad de los Estados de cumplir sus propias obligaciones en materia de derechos humanos, y no disminuye esas obligaciones ${ }^{11}$. Por tanto, aunque no es una norma vinculante, no carece completamente de contenido «normativo». Implica, a su vez, el cumplimiento de las leyes y reglamentos nacionales aplicables en materia de derechos humanos, incluidas las leyes que establecen el due diligence (o diligencia debida) obligatorio en materia de derechos huma-

\footnotetext{
9 Véase Comité de Derechos Económicos, Sociales y Culturales de la ONU, Observación General núm. 24, State Obligations Under the International Covenant on Economic, Social and Cultural Rights in the Context of Business Activities, 2017, párrs. 30-35. Véase también Consejo de Derechos Humanos, Concluding Observations on the Sixth Periodic Report of Germany, Adopted by the Committee at its 106th session (15 Octobe-2 November 2012), 2012, párr. 16.

10 Véase el Principio 9 de los Principios Rectores; véase también el art. 1, párr. 1.3 del art. 73 de la Carta de las Naciones Unidas, de 26 de junio de 1945, BOE núm. 275, de 16 de noviembre de 1990, pp. 33862-33885.

11 Véase el Principio 11 de los Principios Rectores.
} 
nos para las empresas ${ }^{12}$, pero también respetando todos los derechos humanos reconocidos internacionalmente (no solo los derechos civiles y políticos, sino también los derechos económicos, sociales y culturales y las normas fundamentales del trabajo), yendo más allá del cumplimiento de la legislación nacional cuando sea necesario y teniendo en cuenta los derechos de las personas pertenecientes a los grupos más marginados y vulnerables (minorías, pueblos indígenas, defensores de los derechos humanos, etc.) que puedan verse afectados negativamente por sus operaciones.

Dos aspectos cruciales merecen ser destacados aquí. En primer lugar, la necesidad de que las empresas que participan en operaciones de compraventa de tierras respeten las leyes nacionales se ha convertido en un elemento esencial de escrutinio para los órganos de vigilancia de los derechos humanos. Estos últimos no han dudado en abordar directamente al sector empresarial, por ejemplo, con respecto a la situación de los pastores locales que han perdido el acceso a sus tierras de pastoreo tradicionales como consecuencia de la exploración y extracción de minerales, instando a las empresas comerciales que participan en actividades mineras a cumplir con sus obligaciones en virtud de la legislación (nacional) de restaurar la tierra después de la explotación minera ${ }^{13}$.

En segundo lugar, en relación al impacto en los derechos humanos de las actividades relacionadas con la tierra realizadas por la industria extractiva, los organismos y tribunales de derechos humanos han comenzado a aplicar los Principios Rectores y, específicamente, la responsabilidad corporativa de respetar los derechos humanos, reconociendo explícitamente que las empresas no solo deben respetar y proteger los derechos humanos, sino también "prevenir, mitigar y hacerse responsables por las consecuencias negativas de sus actividades sobre los derechos humanos» ${ }^{14}$. Para cumplir con esta responsabilidad, las empresas deben contar con políticas adecuadas a su tamaño y circunstancias, entre las que se incluyen: el compromiso político de asumir su responsabilidad de respetar los derechos humanos; llevar a cabo procesos de vigilancia (due diligence) en materia de derechos humanos para identificar, prevenir, mitigar y rendir cuentas sobre la forma en que abordan sus impactos sobre los derechos humanos; activar procesos que permitan remediar cualquier efecto adverso sobre los derechos humanos que hayan causado o al que hayan contribuido.

En cuanto al compromiso político, debería incluir los relativos a respetar los derechos legítimos de las comunidades a la tierra y a la tenencia de la tierra, incluidos los que se mantienen de manera consuetudinaria y comunal,

12 Un ejemplo notable es la Ley francesa núm. 2017-399, de 2017, que impone a las empresas francesas por encima de un determinado tamaño la obligación de mantenerse vigilantes para evitar los daños medioambientales y a los derechos humanos causados por sus filiales y a través de sus relaciones comerciales.

13 Véase Consejo de Derechos Humanos, Report of the Working Group on the issue of human rights and transnational corporations and other business enterprises, Visit to Mongolia, 2013, párrs. 59-65.

14 Véase Pueblos Kaliña y Lokono c. Surinam, cit., nota 5, párr. 224. 
y su derecho al consentimiento libre, previo e informado. Esos compromisos reflejan el hecho de que, en los acuerdos sobre tierras a gran escala, la tierra y las relaciones con las comunidades son cuestiones importantes en el campo de los derechos humanos. La política debe ser orientada por importantes expertos internos y/o externos, incluidos los expertos en pueblos indígenas y tierras. Por último, los compromisos políticos deben reflejarse en las políticas y procedimientos operativos de la empresa.

En cuanto a la debida diligencia de las empresas en materia de derechos humanos ${ }^{15}$, esta debería incluir los siguientes cuatro componentes básicos: a) evaluación del impacto real y potencial de sus actividades sobre los derechos humanos; $b$ ) integración de las conclusiones y adopción de medidas al respecto; $c$ ) seguimiento de las respuestas, $\mathrm{y} d$ ) comunicación de la forma en que se abordan los efectos ${ }^{16}$. Al poner en práctica el proceso de debida diligencia con relación a las transacciones de tierras, las empresas deben asumir que los riesgos relacionados con esas operaciones, con sus posibles efectos dramáticos en la subsistencia de las personas y las comunidades, son graves (a menos que se demuestre lo contrario). Por consiguiente, esta debería ser una de las cuestiones a las que hay que dar prioridad para una valiosa evaluación de impacto. Además, al llevar a cabo la evaluación de impacto, se debe tener en cuenta como mínimo la concurrencia de factores como los siguientes: débil gobernanza y falta de transparencia; nivel de corrupción percibido; débil gobernanza de la tierra y falta de claridad de los derechos sobre la tierra y la tenencia de la misma; presencia y dimensión de los conflictos sobre la tierra entre las comunidades y las empresas y/o las autoridades; nivel de espacio democrático para que las comunidades y los defensores de los derechos humanos expresen sus preocupaciones; y la existencia de quejas ante los mecanismos correspondientes. No obstante, una consulta significativa a los grupos potencialmente afectados es esencial para evaluar correctamente los impactos adversos y para dar legitimidad y eficacia al proceso de diligencia debida ${ }^{17}$.

En tercer lugar, las empresas deben integrar los hallazgos de las evaluaciones de impacto en todos los procesos y funciones internas pertinentes y tomar las medidas adecuadas. Las medidas adecuadas variarán en función de si la empresa comercial causa o contribuye a un impacto adverso o si solo está vinculada a él. Si la empresa está causando el impacto, debe tomar medidas para detenerlo o prevenirlo; si está contribuyendo al impacto, debe tomar medidas para detener o prevenir su contribución y utilizar el apalancamiento para mitigar el impacto restante; si no ha contribuido al impacto, pero ese impacto está directamente relacionado con sus operaciones, productos o servicios por medio de sus relaciones comerciales, en la mayor medida

15 Véase, en general, Fasciglione, M., «The Enforcement of Corporate Human Rights Due Diligence: From the UN Guiding Principles on Business and Human Rights to the EU Countries Legal System», Human Rights and International Legal Discourse, vol. 10, 2016, núm. 1, pp. 94-117.

16 Véanse los Principios 17 a 21 de los Principios Rectores.

17 Véase el Principio Rector 18. 
posible debe tomar medidas para obtener y utilizar el apalancamiento a fin de prevenir y mitigar el impacto. Sin embargo, el componente de seguimiento es necesario para que una empresa pueda saber si sus políticas de derechos humanos están siendo implementadas y si responden efectivamente a los impactos sobre los derechos humanos. Aquí se pide a las empresas que lleven a cabo procesos inclusivos de consulta y participación de las partes interesadas, incluyendo disposiciones para informar a todos los miembros de la comunidad afectados sobre sus derechos de tenencia.

Cabe señalar que, de conformidad con los principios y normas internacionales en el caso de los pueblos indígenas, la consulta y la participación deben incluir la obtención del consentimiento libre, previo e informado de las comunidades anfitrionas ${ }^{18}$, ya que el respeto de este derecho es una responsabilidad compartida que los Estados y las empresas deben asegurar, en lo que respecta a las empresas, en todas sus operaciones, incluso durante la realización de la debida diligencia. También es importante la comunicación sobre los efectos adversos reales, planteada por las personas y comunidades afectadas, o en su nombre. Al respecto, los Principios Rectores instan a las empresas a que estén preparadas para comunicar los riesgos en materia de derechos humanos que han identificado en sus operaciones y lo que se ha hecho para hacerles frente. La comunicación puede adoptar muchas formas, pero debe ser accesible para el público al que va dirigida, en este caso las comunidades locales ${ }^{19}$.

Por último, en relación con la reparación, tanto los Estados como las compañías deben rendir cuentas de sus acciones que afecten a los derechos humanos en el marco de las prácticas de land grabbing. Las empresas deben tomar medidas para prevenir la corrupción en sus prácticas comerciales, especialmente en relación con la asignación de derechos de tenencia de la tierra. Además, es esencial establecer disposiciones eficaces de vigilancia y aplicación de la ley, incluidos mecanismos apropiados de solución de controversias y de presentación de reclamaciones, para mantener las normas internacionales en esta esfera.

Palabras clave: acaparamiento de tierras, Principios Rectores de las Naciones Unidas sobre las empresas y los derechos humanos, obligaciones positivas, debida diligencia de las empresas en materia de derechos humanos, pueblos indígenas.

Keywords: land grabbing, UN Guiding Principles on business and human rights, positive obligations, corporate human rights due diligence, indigenous peoples.

18 Véanse los arts. 19 y 32 de la Declaración de las Naciones Unidas sobre los derechos de los pueblos indígenas (Asamblea General de la ONU, Resolución 61/295, Declaración de las Naciones Unidas sobre los derechos de los pueblos indígenas, UN Doc. A/61/L.67 y Add.1, de 13 de septiembre de 2007), y los arts. 6, 7 y 15 del Convenio núm. 169 de la OIT (Organización Internacional del Trabajo, Convenio núm. 169 sobre Pueblos Indígenas y Tribales en Países Independientes, de 27 de junio de 1989, BOE núm. 58, de 8 de marzo de 2007, pp. 9923-9928).

19 Véase el Principio Rector 21. 